\title{
Overview of Image Processing and Various Compression Schemes
}

\author{
Akanksha Kumari \\ M.Tech Scholar \\ Department of Electronics and Communication \\ Sagar Institute of Science \& Technology, Bhopal, India
}

\author{
Prof. Kavita kamerikar \\ Assistant Professor \\ Department of Electronics and Communication \\ Sagar Institute of Science \& Technology, Bhopal, India
}

\begin{abstract}
Image processing is key research among researchers. Compression of images are required when need of transmission or storage of images. Demand of multimedia growth, contributes to insufficient bandwidth of network and memory storage device. Advance imaging requires capacity of extensive amounts of digitized information. Therefore data compression is more required for reducing data redundancy to save more hardware space and transmission bandwidth. Various techniques are given for image compression. Some of which are discussed in this paper.
\end{abstract}

Keywords- Image, wavelet, bandwidth, compression.

$* * * * *$

\section{INTRODUCTION}

Image compression, the workmanship and study of diminishing the measure of information required to speak to an image is one of the most helpful and industrially fruitful innovations in the field of advanced image handling. The quantity of images that are compacted and decompressed day by day is stunning and the compression and decompressions themselves are for all intents and purposes imperceptible to the client. Computerized images are broadly utilized in various different applications.

\section{A. Lossy and lossless image compression}

Picture compression may be lossy or lossless. Lossless compression is favored for recorded purposes and much of the time for restorative imaging, specific illustrations, cut craftsmanship, or funnies. Lossy compression techniques, especially when used at low piece rates, present compression ancient pieces. Lossy methods are especially proper for typical pictures, for instance, photos in applications where minor (a portion of the time intangible) loss of devotion is qualified to achieve a liberal abatement in bit rate. Lossy compression that produces immaterial complexities may be called apparently lossless.

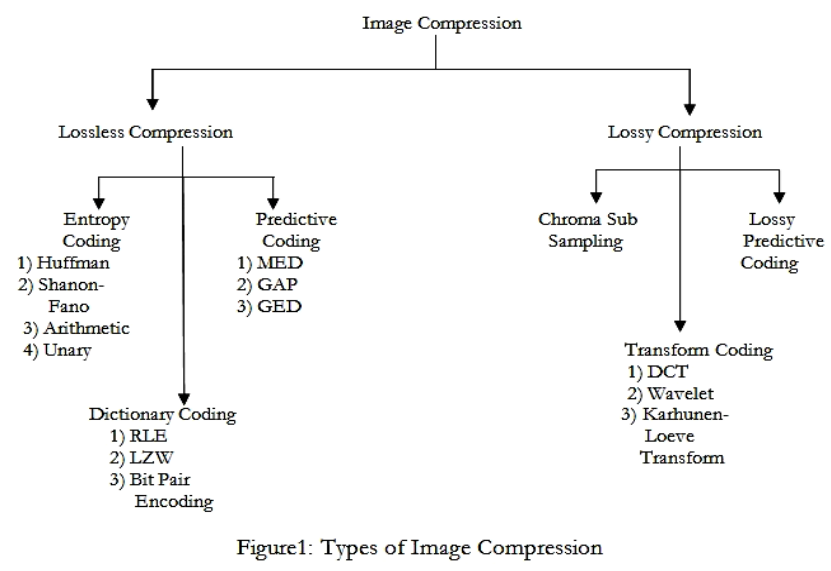

Propelled pictures generally contain gigantic proportions of spatial and spooky abundance. Spatial redundancy is a direct result of the association between's neighboring pixel regards, and powerful abundance is a result of the connection between's different concealing planes. Picture weight (coding) techniques decline the amount of bits required to speak to an image by abusing these redundancies. A contrary system called decompression (unraveling) is associated with the stuffed data to get the changed picture. The objective of weight is to diminish the amount of bits anyway much as could sensibly be normal, while keeping the assurance and the visual idea of the duplicated picture as close to the main picture as could be normal the situation being what it is. This article gives a blueprint of the genuine picture weight techniques. The disentangling adventures for most by far of the coding plans are characteristic and are ordinarily the switch of the encoding steps.

\section{LITERATURE REVIEW}

A. Sengupta et al., [1] A. Sengupta et al., [1] This work proposes two noteworthy oddities. To begin with, it is give a scientific system to equipment asset effective IP center based image compression and decompression (CODEC). The system incorporates CODEC capacities that are equipped for deciding the pixel forces of a packed dark scale image utilizing essentially lesser equipment assets. Advanced pixel estimations of the first image are encouraged as a contribution to the elements of proposed IP system and compacted computerized pixel estimations of packed image created. Thus, computerized pixel estimations of the compacted image are nourished into different elements of the proposed structure for image decompression. Second, the subsequent curiosity is utilizing the determined IP capacities to propose structures of reusable IP centers for complete Haar wavelet transformation (HWT)- based lossy image CODEC. Testing of images from different informational indexes (NASA, therapeutic applications, etc) as far as equipment assets, image quality, 
and compression proficiency have demonstrated that the proposed IP center structure was fruitful in accomplishing equipment proficient CODEC contrasted and JPEG and customary HWT CODECs.

V. S. Thakur et al., [2] The image compression execution of transform coders exceptionally relies upon the energy compaction (EC) ability of transforms. The mainstream transforms, for example, discrete cosine transform (DCT) and discrete wavelet transform (DWT) give tolerable EC; be that as it may, their capacity isn't sufficient to convey great quality image recreation for higher compression levels (CLs). In this investigation, the creators propose another half and half transform which is a combination of wavelet parcel transform (WPT) and square DCT (BDCT) transform to accomplish astounding image compression. This new mixture WPTBDCT transform can achieve higher EC than the current transforms. Further, the creators have discovered another energy compaction file (ECI) to assess the energy minimization of the image transforms. The proposed half and half transform has been widely assessed, based on proposed ECI parameter, the visual quality appraisal of recreated images and with the standard image quality records peak-signal-tonoise ratio and basic comparability file measure.

R. L. de Queiroz et al., [3] In free-perspective video, there is an ongoing pattern to speak to scene questions as solids instead of utilizing various profundity maps. Point mists have been utilized in PC illustrations for quite a while, and with the ongoing plausibility of constant catching and rendering, point mists have been supported over lattices so as to spare calculation. Each point in the cloud is related with its $3 \mathrm{D}$ position and its shading. it is devise a technique to pack the hues in point mists, which is based on a progressive transform and arithmetic coding. The transform is a various leveled subband transform that takes after a versatile variety of a Haar wavelet. The arithmetic encoding of the coefficients expect Laplace appropriations, one for each sub-band. The Laplace parameter for every appropriation is transmitted to the decoder utilizing a custom strategy. The geometry of the point cloud is encoded utilizing the settled octtree examining. Results demonstrate that the proposed arrangement performs similarly with the present best in class, while being considerably more computationally productive. it is accept this work speaks to the best in class in intra-outline compression of point mists for constant 3D video.

P. Cushion et al., [4] The discrete cosine transform (DCT) is known to be asymptotically proportional to the KarhunenLoève transform (KLT) of Gaussian first-request autobackward $(\mathrm{AR}(1))$ forms. Since being uncorrelated under the Gaussian speculation is synonymous with freedom, it likewise yields an autonomous component analysis (ICA) of such signals. In this work, we present a valuable non-Gaussian speculation of this outcome: the portrayal of the ideal symmetrical transform (ICA) for the group of symmetricstable AR(1) forms. The level of sparsity of these procedures is constrained by the steadiness parameter $0<; \quad \alpha \leqslant 2$ with the main non-inadequate individual from the family being the traditional Gaussian $\mathrm{AR}(1)$ process with $\alpha=2$. In particular, it is demonstrate that, for $\alpha<; 2$, a fixed group of operator-like wavelet bases methodicallly beats the DCT as far as compression and denoising capacity. The impact is evaluated with the assistance of two execution criteria (one based on the Kullback-Leibler uniqueness, and the other on Stein's equation for the base estimation blunder) that can likewise be seen as factual proportions of freedom. At last, it is see that, for the sparser sort of procedures with $0<$; $a \leqslant 1$, the operator-like wavelet premise, as managed by direct framework hypothesis, is undistinguishable from the ICA arrangement got through numerical improvement.

J. Lin et al., [5] In this correspondence, another two-channel covered square transform is presented by prefiltering the Haar transform. The prefiltering utilizes plane revolutions with one single turn edge to accomplish high computational effectiveness. The subsequent transform is proportionate to a periodically time-varying (PTV) channel bank. The proposed transform or PTV channel bank has a few focal points for image coding. To begin with, symmetric augmentation is effectively performed at the limits regardless of the way that the channels are unbalanced. Second, arithmetic intricacy can be decreased to basic moves and includes. At last, the presentation for image compression is demonstrated to be high, both as far as visual quality and PSNR. Thus, the proposed transform is a focused option in contrast to the $5 / 3$ and the $9 / 7$ channels, which are as of now well known for subband/wavelet image coders.

A. Golwelkar et al., [6] This work explores 3-D subband coding by means of movement repaid transient sifting (MCTF) utilizing biorthogonal channels. While nonoverlapping Haar channel based MCTF has a fixed size gathering of pictures (GOP) structure, for longer channels it is have to utilize augmentations at the GOP closures bringing about coding proficiency misfortune as well as huge peak signal-to-noise (PSNR) variety. it is take care of this issue by presenting a "sliding window" way to deal with MCTF. While it is locate the more drawn out channels have higher coding gain and critical PSNR improvement at high piece rates, an essential multiplying of the quantity of movement vectors (MVs) causes a drop in PSNR at lower bit rates. The work at that point focuses on improving the productivity of both the MV estimation and compression. it is utilize the MV gauge at higher worldly goals as the beginning stage for nearby MV 
refinement at the present fleeting goals in this way diminishing the multifaceted nature of movement search and getting a progressively uniform movement field. it is improve MV coding execution by adjusting the context-based binary arithmetic coder (CABAC) from H.264. Rather than encoding MV residuals along a quadtree filtering way, it is decrease the MV bit rate by expectation from both neighboring MV squares and squares in the past or the following lower fleeting level. Our proposed movement estimation and coding plans can lessen the MV bit rate by $10 \%-20 \%$ for Haar and $15 \%-30 \%$ for $5 / 3 \mathrm{MCTF}$

C. Tillier et al., [7] Late leaps forward moving repaid fleeting wavelet separating have at long last empowered usage of exceptionally productive versatile and blunder strong video codecs. These new wavelet codecs give various focal points over nonscalable customary arrangements methods based on movement repaid forecast, for example, no recursive prescient circle, separation of noise and examining curios from the substance through utilization of longer fleeting channels, evacuation of long go just as short extend transient redundancies, and so on. Additionally, these wavelet video coding plans can give adaptable spatial, fleeting, signal-tonoise ratio and unpredictability adaptability with fine granularity over a huge scope of bit rates, while keeping up a high coding effectiveness. Nonetheless, most movement remunerated wavelet video plans are based on old style twoband deteriorations that offer just dyadic factors of fleeting versatility.

S. Cheung et al., [8] it is characterize comparative video content as video arrangements with practically indistinguishable substance however perhaps compacted at various characteristics, reformatted to various sizes and edge rates, experienced minor altering in either spatial or transient area, or abridged into keyframe groupings. Building a web crawler to distinguish such comparative substance in the Internet requires: 1) hearty video closeness estimations; 2) quick comparability search methods on huge databases; and 3) natural association of query items. In a past work, we proposed a randomized procedure called the video signature (ViSig) technique for video comparability estimation. In this work,we center around the staying two issues by proposing an element extraction plot for quick likeness search, and a bunching calculation for recognizable proof of comparative groups. Like numerous other substance based techniques, the ViSig strategy utilizes high-dimensional component vectors to speak to video. To warrant a quick reaction time for likeness look on high dimensional vectors, it is propose a novel nonlinear element extraction plot on self-assertive measurement spaces that consolidates the triangle disparity with the traditional Principal Component Analysis (PCA).
Table 1: Summary of literature survey

\begin{tabular}{|c|c|c|c|c|}
\hline $\begin{array}{c}\mathrm{Sr} \\
\mathrm{No}\end{array}$ & $\begin{array}{l}\text { Author } \\
\text { Name }\end{array}$ & $\begin{array}{l}\text { Publish } \\
\text { Details }\end{array}$ & $\begin{array}{c}\text { Proposed } \\
\text { Work }\end{array}$ & Outcome \\
\hline 1 & $\begin{array}{c}\text { A. } \\
\text { Sengu } \\
\text { pta }\end{array}$ & $\begin{array}{c}\text { IEEE, } \\
2018\end{array}$ & $\begin{array}{l}\text { Proposes two } \\
\text { major } \\
\text { novelties. IP } \\
\text { core-based } \\
\text { and CODEC. }\end{array}$ & $\begin{array}{l}\text { Successful } \\
\text { achieving } \\
\text { hardware } \\
\text { efficient } \\
\text { CODEC } \\
\text { compared with } \\
\text { JPEG and } \\
\text { HWT }\end{array}$ \\
\hline 2 & $\begin{array}{c}\text { V.S. } \\
\text { Thakur }\end{array}$ & $\begin{array}{c}\text { IEEE, } \\
2017\end{array}$ & $\begin{array}{l}\text { Hybrid } \\
\text { transform } \\
\text { based on } \\
\text { proposed ECI } \\
\text { parameter }\end{array}$ & $\begin{array}{l}\text { Higher EC and } \\
\text { outperforms } \\
\text { the transforms. }\end{array}$ \\
\hline 3 & $\begin{array}{c}\text { R. L. } \\
\text { de } \\
\text { Queiro } \\
\text { z }\end{array}$ & $\begin{array}{c}\text { IEEE, } \\
2016\end{array}$ & $\begin{array}{l}\text { Hierarchical } \\
\text { transform and } \\
\text { arithmetic } \\
\text { coding. }\end{array}$ & $\begin{array}{l}\text { Much more } \\
\text { computationall } \\
\text { y efficient. }\end{array}$ \\
\hline 4 & P. Pad & $\begin{array}{c}\text { IEEE, } \\
2015\end{array}$ & $\begin{array}{l}\text { Cconstructive } \\
\text { non-Gaussian } \\
\text { generalization }\end{array}$ & $\begin{array}{l}\text { Observe that, } \\
\text { for the sparser } \\
\text { kind } \\
\text { processes with } \\
0<; \alpha \leq 1 \text {. }\end{array}$ \\
\hline 5 & J. Lin & $\begin{array}{c}\text { IEEE, } \\
2010\end{array}$ & $\begin{array}{l}\text { Two-channel } \\
\text { overlapped } \\
\text { block } \\
\text { transform is } \\
\text { introduced by } \\
\text { prefiltering. }\end{array}$ & $\begin{array}{lr}\text { High, } & \text { visual } \\
\text { quality } & \text { and } \\
\text { PSNR. As a } \\
\text { result, }\end{array}$ \\
\hline 6 & $\begin{array}{c}\text { A. } \\
\text { Golwel } \\
\text { kar }\end{array}$ & $\begin{array}{c}\text { IEEE, } \\
2007\end{array}$ & $\begin{array}{l}\text { Non } \\
\text { overlapping } \\
\text { Haar filter- } \\
\text { based MCTF. }\end{array}$ & $\begin{array}{l}\text { Reduce the } \\
\text { MV bit rate by } \\
10 \%-20 \% \text { for } \\
\text { Haar and } 15 \%- \\
30 \% \text { for } 5 / 3 \\
\text { MCTF }\end{array}$ \\
\hline 7 & $\begin{array}{c}\text { C. } \\
\text { Tillier }\end{array}$ & $\begin{array}{c}\text { IEEE, } \\
2006\end{array}$ & $\begin{array}{l}\text { Three-band } \\
\text { temporal } \\
\text { structure that } \\
\text { extends the } \\
\text { concept of } \\
\text { MCTF }\end{array}$ & $\begin{array}{l}\text { Higher } \\
\text { temporal } \\
\text { scalability } \\
\text { flexibility, } \\
\text { improved } \\
\text { compression }\end{array}$ \\
\hline 8 & $\begin{array}{c}\text { S. } \\
\text { Cheun } \\
\text { g }\end{array}$ & $\begin{array}{c}\text { IEEE, } \\
2005\end{array}$ & $\begin{array}{l}\text { Novel } \\
\text { nonlinear } \\
\text { feature } \\
\text { extraction } \\
\text { scheme on } \\
\text { arbitrary } \\
\text { metric spaces. }\end{array}$ & $\begin{array}{l}\text { Outperforms } \\
\text { PCA, Fastmap, } \\
\text { Triangle- } \\
\text { Inequality } \\
\text { Pruning, and } \\
\text { Haar wavelet } \\
\text { on signature. }\end{array}$ \\
\hline
\end{tabular}




\section{HAAR WAVELET TRANSFORM}

The haar wavelet is a succession of rescaled "square-molded" capacities which together frame a wavelet family or premise. Wavelet investigation is like Fourier examination in that it permits an objective capacity over an interim to be spoken to as far as an orthonormal premise. The Haar succession is presently perceived as the primary referred to wavelet premise and broadly utilized as an instructing illustration.

The Haar arrangement was proposed in 1909 by Alfréd Haar. Haar utilized these capacities to give a case of an orthonormal framework for the space of square-integrable capacities on the unit interim $[0,1]$. The investigation of wavelets, and even the expression "wavelet", did not come until some other time. As a unique instance of the Daubechies wavelet, the Haar wavelet is otherwise called $\mathrm{Db} 1$.

The Haar wavelet is likewise the easiest conceivable wavelet. The specialized weakness of the Haar wavelet is that it isn't ceaseless, and consequently not differentiable. This property can, be that as it may, be preference for the investigation of signs with sudden advances, for example, checking of hardware disappointment in machines.

\section{CONCLUSION}

In this paper we discuss about the image processing and compression approaches of previous work. Haar and wavelet transform approach used by many authors to achieve higher compression ratio. This paper discusses about various existing image compression techniques. They can be classified mainly to lossless or near-lossless compression techniques. Lossless compression techniques can achieve only low compression ratio and hence near-lossless techniques are used to compress medical images with tolerable level of loss of information.

\section{REFERENCE}

[1]. A. Sengupta, D. Roy, S. P. Mohanty and P. Corcoran, "A Framework for Hardware Efficient Reusable IP Core for Grayscale Image CODEC," in IEEE Access, vol. 6, pp. 871$882,2018$.

[2]. V. S. Thakur, S. Gupta and K. Thakur, "Hybrid WPTBDCT transform for high-quality image compression," in IET Image Processing, vol. 11, no. 10, pp. 899-909, 10 2017.

[3]. R. L. de Queiroz and P. A. Chou, "Compression of 3D Point Clouds Using a Region-Adaptive Hierarchical Transform," in IEEE Transactions on Image Processing, vol. 25, no. 8, pp. 3947-3956, Aug. 2016.

[4]. P. Pad and M. Unser, "Optimality of Operator-Like Wavelets for Representing Sparse AR(1) Processes," in IEEE Transactions on Signal Processing, vol. 63, no. 18, pp. 4827-4837, Sept.15, 2015.
[5]. J. Lin and M. J. T. Smith, "A Two-Channel Overlapped Block Transform for Image Compression," in IEEE Transactions on Image Processing, vol. 19, no. 11, pp. 3064-3071, Nov. 2010.

[6]. A. Golwelkar and J. W. Woods, "Motion-Compensated Temporal Filtering and Motion Vector Coding Using Biorthogonal Filters," in IEEE Transactions on Circuits and Systems for Video Technology, vol. 17, no. 4, pp. 417 428, April 2007.

[7]. C. Tillier, B. Pesquet-Popescu and M. van der Schaar, "3band motion-compensated temporal structures for scalable video coding," in IEEE Transactions on Image Processing, vol. 15, no. 9, pp. 2545-2557, Sept. 2006.

[8]. S. -. Cheung and A. Zakhor, "Fast similarity search and clustering of video sequences on the world-wide-web," in IEEE Transactions on Multimedia, vol. 7, no. 3, pp. 524537, June 2005

[9]. S.-C. Cheung, A. Zakhor, "Efficient video similarity measurement with video signature", IEEE Trans. Circuits Syst. Video Technol., vol. 13, no. 1, pp. 59-74, Jan. 2003.

[10].S.-C. Cheung, Efficient Video Similarity Measurement and Search, 2002 